\section{DEVELOPMENT OF A DECISION BOX OF DIAGNOSTIC SYSTEM FOR ELECTRIC DRIVES}

\section{PAVEL STEPANOV ${ }^{1}$, PAVOL BOZEK ${ }^{2}$, STANISLAV LAGUTKIN ${ }^{3}$, YURY NIKITIN ${ }^{4 *}$}

${ }^{1}$ National Research Nuclear University MEPhl, Department of Control Automation, Novouralsk, Sverdlovsk Region, Russia

${ }^{2}$ Slovak University of Technology in Bratislava, Faculty of Materials Science and Technology, Institute of Production

Technologies, Slovakia

${ }^{3}$ National Research Nuclear University MEPhl, Department of Mechanical Engineering Technology, Novouralsk, Sverdlovsk Region, Russia

${ }^{4}$ Kalashnikov Izhevsk State Technical University, Department of Mechatronic Systems, Izhevsk, Russia

DOI: 10.17973/MMSJ.2021_11_2021126 e-mail: nikitin@istu.ru

The paper describes a diagnostic system for electromechanical equipment with a decision-making block based on a neural network. An asynchronous gear drive was used as a control object. Decision making was carried out on the basis of a comprehensive analysis of vibration data (from the gear drive) and the current consumption of the induction motor. Vibration velocity, vibration acceleration and current in the phases of the stator winding of the drive electric motor are distinguished as diagnostic signs. The work shows the possibility of increasing the efficiency of diagnostics of electromechanical equipment by using complex analysis with the use of an intelligent decision-making unit. Analysis of the results of the neural network operation with the received vibration and current data showed that with a smaller number of iterations (training time) (by $40 \%), 97.9 \%$ of correct answers and a lower error value (by $12 \%$ ) were obtained.

KEYWORDS

Current, vibration, neural network, diagnostics, electric drive.

\section{INTRODUCTION}

With the increasing automation of modern production (Industry 4.0), the requirements for its reliability are increasing. Therefore, diagnostics (control of technical condition) of equipment is becoming the most promising and rapidly developing aspect of modern mechanical engineering. At present, the theoretical foundations for assessing the technical state of electric drive systems are insufficiently developed due to the complexity of the physical processes occurring in such systems, the complexity of the mathematical formalization of the description of these processes and defects, with the limited measured diagnostic parameters, with measurement errors. Due to the above-mentioned reasons, there are no descriptions of the regularities between the diagnostic parameters and the states of electric drives. Therefore, it is not possible to systematically solve the problems of diagnosing and assessing the residual resource, planning maintenance and repair.

In industry, an asynchronous electric drive is widely used due to a number of advantages: high reliability, low cost. In engineering practice, it is the use in the field of production technology and production technologies such as turning, milling, drilling, etc. [Kolesnyk 2020, Sentyakov 2020, Peterka 2013, Vopat 2014]. The main purpose of the research is to improve the efficiency of diagnosing the states of an electric drive through the use of an integrated approach based on the analysis of information of different physical nature (vibration and current consumption) generated by individual drive elements. In an electric drive, electrical processes occur, which are characterized by electric current, and mechanical processes, which are characterized by vibration. Therefore, the choice of these diagnostic parameters is obvious.

In work [Stepanov 2014] such diagnostic features as the coefficients of the wavelet transformation of vibration (using the example of vibration velocity) and the current consumed by the drive motor are investigated. As a supplement, the vibration velocity spectra are also considered

The modern level of development of hardware and software allows high-quality data collection and processing, as well as displaying information and making decisions about the state of the equipment. One of the promising directions in the development of means for monitoring the technical condition of equipment is the use of neural networks [Stepanov 2013].

The detection and diagnosis of motor faults based on a metacognitive network of random vector functional relationships is discussed in [Sayed-Mouchaweh 2018]. The book [Saad 2019] covers various issues related to motor condition monitoring, signal processing and conditioning, instrumentation and measurements, faults for induction motors failures, new trends in condition monitoring, and the fault identification process using 
motor currents electrical signature analysis. For detection of the various faults usually affecting motors, several techniques have been proposed and used successfully [Isermann 2006, Murcinkova 2013, Costa 2016]. However, a good understanding of the mechanical and electrical properties of the motor in healthy and faulty conditions significantly influences the accuracy and reliability of the online condition monitoring methods.

Methods of diagnostics on fuzzy logic and neural networks are considered in [Kuric 2021, Bozek 2021, Nikitin 2020a]. It is shown that these methods give good results for finding defects in motors. The various issues of diagnostics of electric motors are considered in [Peterka 2020, Turygin 2018, Thomson 2017, Nikitin 2020b, Lekomtsev 2021]. Diagnostic methods based on the current signature analysis of the electric motor are discussed in [Luo 2017, Qiu 2020]. The diagnosis of bearing in electric motors is discussed in [Ojaghi 2018, Cui 2017]. Fault-Tolerant electrical machines and drives is discussed in [Mustafa 2017]. The pioneer studies of such systems are fault diagnosis studies.

On the basis of existing research, modern methods of diagnostics of the technical condition of electromechanical equipment have been studied. The standards for vibration control are also considered. It was revealed that the following trends can be traced in the field of diagnostics:

1. Development has received the diagnosis of electric drives only for one type of diagnostic parameters (vibration of equipment) without taking into account the interaction of electrical and mechanical elements of the equipment.

2. Most modern diagnostic systems have the ability to collect signals from additional sensors (temperature, current, etc.) in addition to vibration sensors. However, their use is limited only to the output of the overall level of such a signal.

3. At the same time, there is a need to improve the efficiency of diagnostics and to eliminate errors and false alarms by introducing an integral (complex) assessment of the technical condition.

The authors concluded that the analysis of vibration, as the most common type of diagnostic parameters, in some cases may not be enough. One of such cases is diagnostics of electric drives. For these units, the necessity of the complex use of mechanical and electrical parameters has been substantiated.

Thus, in this work, the task is to improve the efficiency of the diagnostic process for electric drives using an intelligent decision-making unit based on vibration and current sensors.

\section{LABORATORY BENCH AND RECORDING EQUIPMENT}

A laboratory stand (asynchronous drive with a worm gear, Fig. 1) was used as an object of diagnostics. The power of the asynchronous electric motor is $P=0.18 \mathrm{~kW}$. Rotation speed $n=1350 \mathrm{rpm}$. Worm gear MCh-40M-31.5-47.6-515-1S-U3. Load on the output shaft of the worm gear $M=$ $32 \mathrm{~N} \cdot \mathrm{m}$. In laboratory conditions, the following malfunctions were identified and reproduced: reduction of the contact patch of the gear transmission, misalignment of the gear transmission, grazing in the engagement zone, grazing on the drive motor shaft, rotor imbalance, loosening of the foundation (fastening), lack of lubrication.

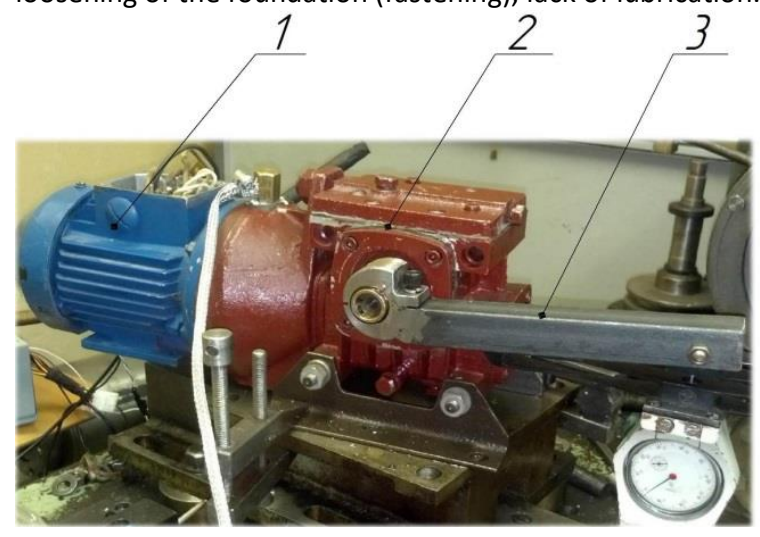

Figure 1. Laboratory bench: 1 - asynchronous motor; 2 - worm gear; 3 - loading device

To obtain diagnostic information at both stands, the following sensors were used: vibration transducer AR2019, current sensors LEM LA-55P.

Tab. 1 and Tab. 2 shows the technical parameters of the sensors used: vibration transducer AR2019 and current sensors LEM LA-55P.

\begin{tabular}{|l|l|}
\hline $\begin{array}{l}\text { Parameter of Vibration transducer } \\
\text { AR2019 }\end{array}$ & Value \\
\hline Noise level, RMS $(1 \mathrm{~Hz}-10 \mathrm{kHz}), \mathrm{g}$ & $<0.005$ \\
\hline $\begin{array}{l}\text { Frequency response (flatness } \pm 1 \mathrm{~dB}), \\
\mathrm{Hz}\end{array}$ & $5-30000$ \\
\hline Working temperature range, ${ }^{\circ} \mathrm{C}$ & $-40 \ldots+125$ \\
\hline Maximum impact (peak value), g & \pm 10000 \\
\hline Amplitude range, g & \pm 7000 \\
\hline Transverse sensitivity & $<5 \%$ \\
\hline Axial sensitivity, $\mathrm{mV} / \mathrm{g}$ & $0.5 \pm 10 \%$ \\
\hline
\end{tabular}

Table 1. Technical parameters of the AR2019 vibration transducer

\begin{tabular}{l|l|}
\hline $\begin{array}{l}\text { Parameter of LEM LA-55P current } \\
\text { sensor }\end{array}$ & Value \\
\hline Rated value of the measured current, A & 50 \\
\hline Response time, ns & 50 \\
\hline Output, mA & 25 \\
\hline
\end{tabular}




\begin{tabular}{|l|l|}
\hline Measurement range, $\mathrm{A}$ & \pm 100 \\
\hline Bandwidth, $\mathrm{kHz}$ & 200 \\
\hline Working voltage, $\mathrm{V}$ & $\pm 15( \pm 5 \%)$ \\
\hline $\begin{array}{l}\text { Working temperature, }{ }^{\circ} \mathrm{C} \\
\text { Accuracy, at rated current in the } \\
\text { primary winding, }\end{array}$ & $-40 \ldots+85$ \\
$\mathrm{~T}=25{ }^{\circ} \mathrm{C}, \pm 15 \mathrm{~V}( \pm 5 \%)$ - power supply & \\
\hline
\end{tabular}

Table 2. Technical parameters of the AR2019 vibration transducer and the LEM LA-55P current sensor

The current sensors were installed in the electric motor control unit (for two phases), and the vibration sensor was installed at the control point on the gear reducer in accordance with GOST R ISO 13373-1-2009.

Vibration transducer AR-2019 needs external power supply. The connection diagram of the sensor to the equipment and the matching device is shown in Figures 2 and 3.

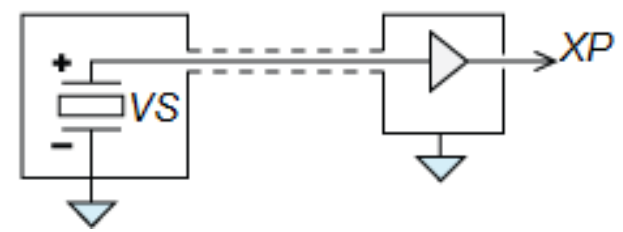

Figure 2. Connection diagram of the AP2019 to the recording equipment: VS - vibration transducer, XP - output from the matching device

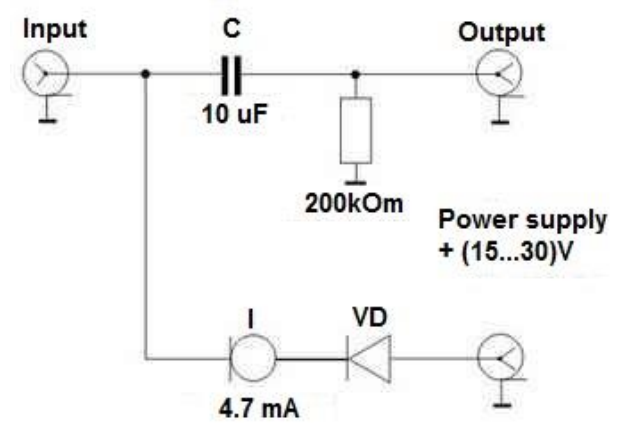

Figure 3. Diagram of the matching device for the AP2019 sensor

The terminator output connects to the NI SCB-68 connector block. LEM LA-55P current sensors are installed on the stator windings of the drive motor. The sensor circuit and its connection are shown in Figure 4.

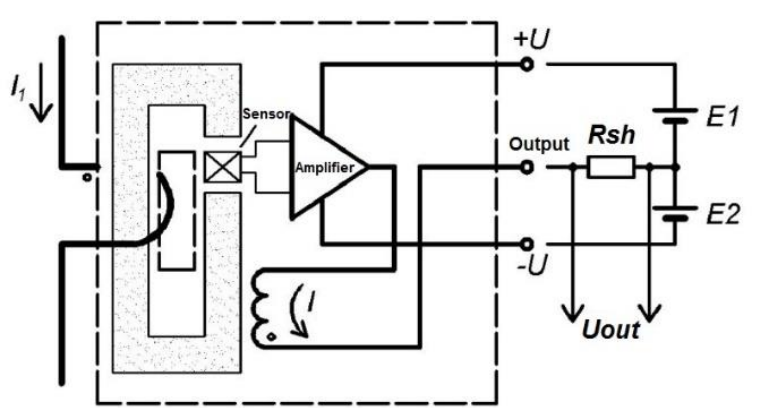

Figure 4. LEM LA-55P sensor connection diagram

A resistor of $100 \mathrm{Ohm}$ is selected as Rsh.

The calculation of the coefficients of the discrete wavelet transform was carried out using the developed software in the LabView environment (data collection and processing unit). The reliability of the calculation was confirmed in the MatLab software environment using the Wavelet Toolbox.

\section{DEVELOPMENT OF THE ARCHITECTURE OF THE DECISION BOX}

As part of the research, work was carried out to select the optimal architecture of the neural network classifier and it was concluded that the best results in training and testing are achieved precisely on the basis of a 3-layer neural network with the number of neurons on the input layer 80 , on the intermediate layer -1000 and on the output 8.

Based on the application of the basic principles of building decision-making systems, a model for recognizing the technical states of electromechanical equipment has been developed on the basis of a 3-layer neural network classifier (Fig. 5) with a nonlinear activation function of computational elements in a layer (bipolar sigmoid) and a learning algorithm based on backpropagation based error. The proposed model is capable of developing a decision on the state of the object for various combinations of diagnostic features that were not previously encountered in the training sample, and thereby increasing the reliability of recognizing the technical condition of the equipment.

The developed architecture includes an input layer (80 neurons, labeled $\left.X_{1}, \ldots, X_{i}, \ldots, X_{n}\right)$, an intermediate layer (1000 neurons, labeled $Z_{1}, \ldots, Z_{j}, \ldots, Z_{m}$ ) and an output layer (8 neurons, labeled $Y_{1}, \ldots, Y_{k}, \ldots, Y_{p}$ ). The input layer receives information about the current state of diagnostic features (the number of neurons is equal to the number of diagnostic features), data is processed on the intermediate layer, and a larger number of neurons ultimately lead to more accurate results and a decrease in the performance of the network as a whole. At the output layer, decisions about the state of the object are issued (the number of 
neurons is equal to the number of recognizable states of the equipment).

Neurons representing the network outputs (designated $Y_{1}, \ldots, Y_{k}, \ldots, Y p$ ) and hidden neurons can have an offset of 1 (Fig. 5). These biases serve as weights on the connections $(v, w)$ emanating from neurons, the output of which always appears 1 . During the learning process, the signals propagate in the opposite direction, where the network response error is calculated and the weighting coefficients $v$ and $w$. The output signal $Y$ takes on a maximum value in\%, which corresponds to a specific technical condition of the equipment (the probability of this condition).

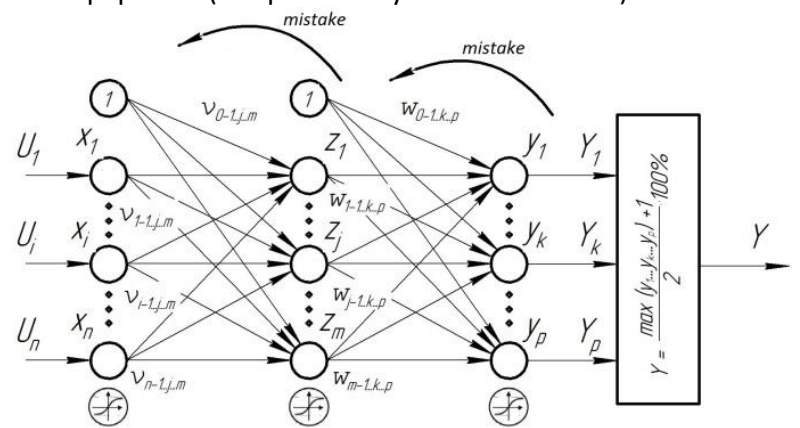

Figure 5. Neural network architecture

The decision box was implemented as a separate environment with the ability to load data from the data collection subsystem.

The training was built on the basis of the error back propagation algorithm (Fig. 5).

On one laboratory bench in each experiment, a certain type of defect or malfunction was created by changing either the position of the worm wheel or replacing it ( 5 wheels were made). After testing with a gear reducer, the reducer worm wheel was replaced (5 gear wheels were replaced). In each experiment, about 1000 measurements were carried out.

\section{DECISION BOX TRAINING}

Training and testing of the developed network was carried out on the basis of experimental data obtained at the laboratory bench when simulating defects. The training sample included 20 features (general level and Daubechies wavelet coefficients $\mathrm{db}-8$ - root-mean-square values (hereinafter - RMS) and their maximum values (max)) for each information flow (vibration acceleration, vibration velocity and two electric current of phases), supplemented with a noise component $(10-200 \%$ of the standard deviation (hereinafter - SD) for vibration acceleration and vibration velocity and $10-300 \%$ of SD for current). With such a sample, the neural network was trained (about 40000 iterations), the result of which is shown in Tab. 3.

\begin{tabular}{|l|l|}
\hline Parameter & Value \\
\hline Number of hidden layers & 1 \\
\hline $\begin{array}{l}\text { The number of neurons on the hidden } \\
\text { layer }\end{array}$ & 1000 \\
\hline Vibration noise, \% of RMS & 200 \\
\hline Noise current, \% of RMS & 300 \\
\hline Total iterations & 40473 \\
\hline Total correct answers & 39674 \\
\hline Total errors & 799 \\
\hline Percentage of correct answers, \% & 98 \\
\hline Mean square error & 0.071 \\
\hline Number of neurons on the input layer & 80 \\
\hline The number of neurons on the output layer & 8 \\
\hline
\end{tabular}

Table 3. Neural network training results

Also, a statistical estimate of the root mean square error (MSE) was carried out, defined as the mean square value of the differences between the desired output value ti and the values $Y i$ actually obtained at the network outputs for each example $\mathrm{i}$, averaged over $\mathrm{n}$ tests. Formula (1) characterizes this error:

$M S E=\sqrt{\frac{\sum_{i=1}^{n}\left(t_{i}-Y_{i}\right)^{2}}{n}}$,

where ti is the required response from the neural network; $\mathrm{Yi}$ is the answer received as a result of the neural network; $\mathrm{n}$ is the total number of neural network tests.

The noise component during training affects the sensitivity of the neural network as a whole. The selected noise values ultimately affect the ultimate reliability of the network's responses, as well as the learning rate. For example, in the case of "rotor imbalance" malfunction, the RMS of the wavelet coefficient $d 6$ of the current of the first phase was 0.682 with an RMS of 0.003 . By increasing the RMS value by $300 \%$ (adding a noise component), the network sensitivity also changes (downward), but the recognition accuracy increases (during testing). However, this approach yields good results only with a small number of recognizable output states. In the context of this work, an increase in the noise component is acceptable.

To further substantiate the presence of a connection between mechanical and electrical parameters, we present the results of training a neural network (of the same architecture: one hidden layer with 1000 neurons) when only vibrational (Tab. 4) and only current parameters are supplied to its input (Tab. 5).

It can be seen from the results obtained that this architecture of the neural network shows good results during training when only vibration data is supplied to the 
input (Tab. 4). However, when comparing these learning results with the results obtained on the parameters of vibration and current (Tab. 3), we can conclude that with a smaller number of iterations (training time), we get approximately the same percentage of correct answers and a smaller error value.

\begin{tabular}{|l|l|}
\hline Parameter & Value \\
\hline Vibration noise, \% of RMS & 200 \\
\hline Total iterations & 64487 \\
\hline Total correct answers & 62941 \\
\hline Total errors & 1546 \\
\hline Percentage of correct answers, \% & 97.6 \\
\hline Mean square error & 0.081 \\
\hline
\end{tabular}

Table 4. Results of training the neural network when the parameters of vibration acceleration and vibration velocity are fed into the input

\begin{tabular}{|l|l|}
\hline Parameter & Value \\
\hline Noise current, \% of RMS & 300 \\
\hline Total iterations & 1114867 \\
\hline Total correct answers & 897312 \\
\hline Total errors & 217555 \\
\hline Percentage of correct answers, \% & 80.5 \\
\hline Mean square error & 0.5 \\
\hline
\end{tabular}

Table 5. The results of training the neural network when the current parameters are fed to the input (phase 1 and phase 2)

In fig. 6 shows the effectiveness of training an artificial neural network using various input data: "all data" hereinafter - SD both vibration (vibration acceleration and vibration velocity) and current data (phase 1 current, phase 2 current) are fed to the neural network input.

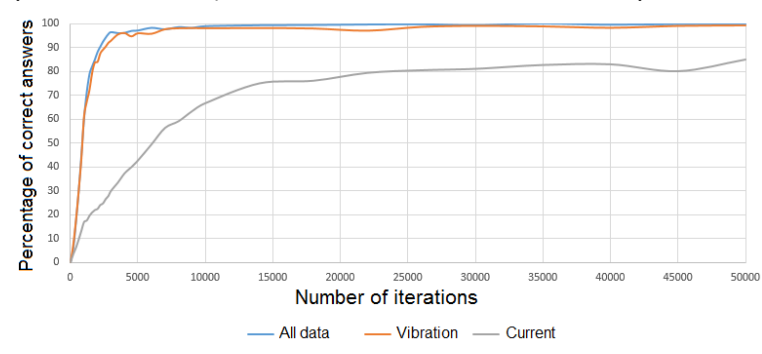

Figure 6. The effectiveness of training an artificial neural network

It can be seen from the presented graph that when only current data is supplied to the input, the training efficiency drops sharply. However, if we compare training with the use of only vibration data, with training with complex data, we can conclude that when the values are $70 \%$ or more, the current parameters have a significant effect on the training duration.
Consequently, the training of the neural network is carried out most efficiently with an increase in the number of input parameters. The positive dynamics during training can also be explained by the presence of the same reaction of the controlled parameters of vibration and current to the occurrence of defects and malfunctions.

\section{DECISION BOX TESTING}

When testing the trained network, the values of diagnostic signs ( 80 values) obtained in laboratory conditions were fed into the input. The test results are shown in table 6. A change in the noise component of the training sample strongly affects the final reliability of the network solutions. The selected value for this component led to the best test results (average confidence about $99 \%$ ).

An important result when testing a neural network was the identification of diagnostic features (wavelet coefficients), which are the most sensitive (informative) to changes in the technical state of electrical equipment. In the studies carried out, the value of the selected controlled traits has been substantiated.

\begin{tabular}{|c|c|c|}
\hline Input data & $\begin{array}{l}\text { Number } \\
\text { of tests }\end{array}$ & $\begin{array}{l}\text { Validity of } \\
\text { decisions, \% }\end{array}$ \\
\hline Working condition & 2121 & 100 \\
\hline $\begin{array}{l}\text { Reduction of the contact patch } \\
\text { of the gear train }\end{array}$ & 1067 & 99.6 \\
\hline Gear misalignment & 1067 & 98.9 \\
\hline $\begin{array}{l}\text { Grazing in the engagement } \\
\text { zone }\end{array}$ & 1063 & 100 \\
\hline $\begin{array}{l}\text { Grazing on the asynchronous } \\
\text { motor shaft }\end{array}$ & 1062 & 100 \\
\hline Rotor imbalance & 1063 & 100 \\
\hline Looseness of fastening & 1056 & 100 \\
\hline Lack of lubrication & 1137 & 97.9 \\
\hline
\end{tabular}

Table 6. Results of testing the neural network after training

In view of the fact that the training set included 20 features supplemented by a noise component of 10-200\% of the standard deviation for vibration acceleration and vibration velocity and $10-300 \%$ of the standard deviation for the current, in table 5 the best results in comparison with table 2-4 were obtained. This manipulation allows to increase the range of diagnostic signs for troubleshooting. In the future, with an increase in the number of faults, it is necessary to adjust the training sample in order to achieve better results during testing.

If a signal is sent to one of the neurons of the output layer and pass it through all the hidden layers to the input layer, the numerical characteristics of the input neurons in relation to this output neuron is given. This allows, as a 
result of the work of the decision-making unit, to select the most sensitive diagnostic signs for assessing the technical condition with the level of confidence in these results (in \%).

For example, for the diagnosis "Lack of gear lubrication" (Fig. 7), the most informative are the wavelet coefficients $\mathrm{a} 8, \mathrm{~d} 5, \mathrm{~d} 2$ and the peak values of the general level and coefficients $d 7, d 6$ of vibration acceleration (99-100 \%), as well as general level, coefficients $d 6, d 5, d 4$, peak values of the general level and coefficients $d 6, d 5, d 4$ of the current of the first phase (99-100 \%).

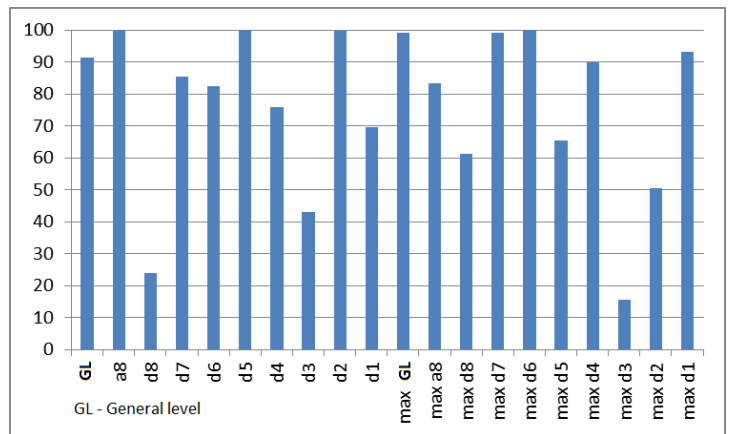

(a)

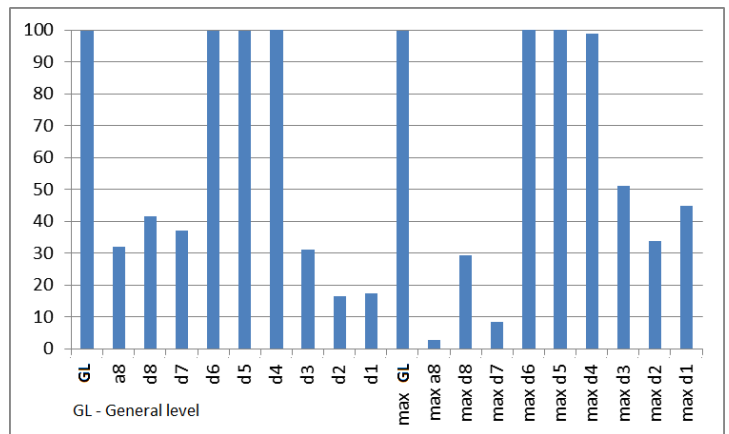

(b)

Figure 7. An example of the selection of sensitive (the level of confidence is indicated on the ordinate axis, in \%) diagnostic signs in the absence of lubrication of the gear transmission in the decision block: a) from the vibration acceleration signal, b) from the current signal of the first phase

\section{CONCLUSIONS}

As a result of the analysis, a method was developed for selecting the most informative diagnostic signs for diagnosing electric drives with a reliability level of results close to $100 \%$, obtained from the output of the decision box. The architecture of a neural network has been developed, which consists of 80 input neurons, 1000 intermediate and 8 output neurons. Analysis of the results of the neural network operation with the obtained vibration and current data showed that with a smaller number of iterations (training time) (by $40 \%$ ), approximately the same percentage of correct answers and a lower error value (by $12 \%$ ) were obtained.

Thus, on the basis of the study carried out, the urgent scientific and technical problem of developing a decisionmaking block, characterized by a joint analysis of mechanical and electrical parameters, has been solved. Thus, the efficiency of the technical condition monitoring is increased in the form of an increase in the reliability indicator up to $97.9 \%$.

For automated processing of experimental research results and subsequent practical implementation, a unified information and measurement system has been developed, which includes a hardware platform and software products for collecting and processing data, as well as a decision-making unit based on a neural network, including a laboratory stand for research malfunctions of the electric drive

The results of the study showed the possibility of increasing the efficiency of diagnostics through the use of an integrated approach (increasing the number of diagnostic signs), which speeds up the adjustment (by 1.5 times), reduces the magnitude of the error and increases the reliability of the decisions.

Thus, based on the analysis of the wavelet coefficients of current and vibration acceleration, reliable detection of such defects as defects of the mechanism as a whole (loosening of fastening and grazing), malfunctions of motors (imbalance of the rotor of an induction motor, grazing of the rotor), malfunctions of the gear transmission (misalignment, reduction of the contact patch, lack of lubrication) is provided. This paper shows the possibility of increasing the efficiency of diagnosing an asynchronous electric drive by using complex analysis using an intelligent decision-making unit.

\section{ACKNOWLEDGMENTS}

The reported study was supported by research project VEGA $1 / 0019 / 20$ and KEGA 006STU-4/2021 by the Slovak Research and Development Agency.

\section{REFERENCES}

[Bozek 2021] Bozek, P. et al. Diagnostics of Mechatronic Systems. Series: Studies in Systems, Decision and Control, Springer Nature, Switzerland AG, 2021, Vol. 345, 160 p. ISBN 978-3-030-67055-9.

[Costa 2016] Costa, B.S.J. Fuzzy Fault Detection and Diagnosis. Handbook on Computational Intelligence. Vol. 1: Fuzzy Logic, Systems, Artifical Neural Networks and Lerning Systems. World Scientific Publishing Co. Pte. Ltd., 2016, pp. 251-288. 
[Cui 2017] Cui, L.L. et al. Quantitative and localization diagnosis of a defective ball bearing based on vertical-horizontal synchronization signal analysis. IEEE Trans. Ind. Electron., 2017, Vol. 64, pp. 8695-8706.

[Isermann 2006] Isermann, R. Fault-Diagnosis Systems: An Introduction from Fault Detection to Fault Tolerance. Berlin; New York, Springer, 2006, $475 \mathrm{p}$.

[Kolesnyk 2020] Kolesnyk, V. et. al. Experiemntal Study of Drilling Temperature, Geometrical Errors and Thermal Expansion of Drill on Hole Accuracy When Drilling CFRP/Ti Alloy Stacks. Materials, 2020, Vol. 13, No. 14, 3232; https://doi.org/10.3390/ma13143232.

[Kuric 2021] Kuric, I. et al. Analysis of diagnostic methods and energy of production systems drives. Processes, 2021, Vol. 9, p. 843.

[Lekomtsev 2021] Lekomtsev P.V., et al. DC Motor Identification Based on Quasi-Optimal Nonlinear Control Algorithm. Vestnik IzhGTU imeni M.T. Kalashnikova, 2021, Vol. 24, No. 2, pp. 68-76 (in Russian). DOI: 10.22213/24131172-2021-2-68-76. ISSN 1813-7903.

[Luo 2017] Luo, H. Plug-and-Play Monitoring and Performance Optimization for Industrial Automation Processes. Springer Fachmedien Wiesbaden $\mathrm{GmbH}, 2017,158 \mathrm{p}$.

[Murcinkova 2013] Murcinkova, Z., Krenicky, T. Applications utilizing the damping of composite microstructures for mechanisms of production machines and manipulator devices. In: SGEM 2013: 13th Int. Multidisciplinary Sci. Geoconf. Vol. 1: 16-22 June, 2013, Albena, Bulgaria. Sofia: STEF92 Technology, 2013. pp. 23-30.

[Mustafa 2017] Mustafa, D., Muhammet, U. (Eds.) Fault Diagnosis and Detection. InTechOpen, 2017. 334 p. ISBN-13 978-953-51-3203-5.

[Nikitin 2020a] Nikitin, Y.R. et al. Logical-linguistic Model of Diagnostics of Electric Drivers with Sensors Support. Sensors, 2020, Vol. 20, Iss. 1, pp. 1-19.

[Nikitin 2020b] Nikitin Y.R. et al. Influence of Diagnostic Stepper Motor Parameters on Criterion of Identifiability for Nonlinear Discrete Model by State Space. Vestnik IzhGTU imeni M.T. Kalashnikova, 2020, vol. 23, no. 4, pp. 52-59 (in Russ.). DOI: 10.22213/2413-1172-2020-4-5259. ISSN 1813-7903.

[Ojaghi 2018] Ojaghi, M. et al. Analytic model for induction motors under localized bearing faults. IEEE Trans. Energy Convers., 2018, Vol. 33, pp. 617-626.

[Peterka 2013] Peterka, J. and Pokorny, P. Influence of the Lead Angle from the Vertical Axis Milling on Radius of the Cutter. In: Proc. $7^{\text {th }}$ Int. Congress of Precision Machining (ICPM 2013), 03-05 Oct. 2013, Miskolc, Hungary, 2013, pp. 44-49.

[Peterka 2020] Peterka, J. et al. Diagnostics of automated technological devices. MM Science Journal, 2020, No. Oct., pp. 4027-4034.

[Qiu 2020] Qiu, C. et al. An Approximate Estimation Approach of Fault Size for Spalled Ball Bearing in Induction Motor by Tracking Multiple Vibration Frequencies in Current. Sensors, 2020, Vol. 20, 1631.

[Saad 2019] Saad, N., et al. Condition Monitoring and Faults Diagnosis of Induction Motors: Electrical Signature Analysis. CRC Press, 2019, 170 p. ISBN 978-0815389957.

[Sayed-Mouchaweh 2018] Sayed-Mouchaweh, M. (Ed.) Fault Diagnosis of Hybrid Dynamic and Complex Systems. Springer International Publishing AG, 2018, 290 p. ISBN 331974013X.

[Sentyakov 2020] Senytyakov, K. et. al. Modeling of Boring Mandrel Working Process with Vibration Damper. Materials, 2020, Vol. 13, No. 8, 1931. DOI10.3390/ma13081931.

[Stepanov 2013] Stepanov, P. and Nikitin Y. Diagnostics of Mechatronic Systems on the Basis of Neural Networks with High-Performance Data Collection. In: Proc. of Mechatronics 2013: Recent Technological and Scientific Advances. Springer Int. Publishing Swizerland, 2014. 7-9 Oct. 2013, Brno, Czech Republic. Springer, pp. 433-440. ISBN 978-3-319-02293-2.

[Stepanov 2014] Stepanov, P. et al. Research of Electric and Mechanical Diagnostic Parameters of Drive Equipment. Applied Mechanics and Materials, 2014, Vol. 683, pp. 177-182.

[Thomson 2017] Thomson W. and Culbert I. Current Signature Analysis for Condition Monitoring of Cage Induction Motors: Industrial Application and Case Histories. Wiley-IEEE Press, 2017, 427 p. ISBN: 978-1-119-02959-5.

[Turygin 2018] Turygin, Y. et al. Reliability Determination and Diagnostics of a Mechatronic System. Advances in Science and Technology, 2018, Vol. 12, pp. 274-290.

[Vopat 2014] Vopat, T. et. al. The Wear Measurement Process of Ball Nose end Mill in the Copy Milling Operations. In: 24th DAAAM International Symposium on Intelligent 
Manufacturing and Automation, 2013, Vol. 69, pp. 1038-1047.

\section{CONTACTS:}

Pavel Stepanov, Ph.D., Assoc. prof.

National Research Nuclear University MEPhl, Department of Control Automation

85, Lenina st., Novouralsk, Sverdlovsk Region, Russia, 624130,push-tet@mail.ru

Pavol Bozek, Ph.D., Prof.

Slovak University of Technology in Bratislava, Faculty of Materials Science and Technology, Institute of Production Technologies, Slovakia, pavol.bozek@stuba.sk

Stanislav Lagutkin, Ph.D., Assoc. prof.

National Research Nuclear University MEPhl, National Research Nuclear University MEPhl, Department of Mechanical Engineering Technology

85, Lenina st., Novouralsk, Sverdlovsk Region, Russia, 624130, lagutkin@nsti.ru

Yury Nikitin, Ph.D., Assoc. prof.

Kalashnikov Izhevsk State Technical University, Department of Mechatronic Systems, 7, Studencheskaya st, Izhevsk, Russia, 426069, nikitin@istu.ru 